\section{A case of curatively resected gastric wall implanta- tion of pancreatic cancer caused by endoscopic ultrasound-guided fine-needle aspiration}

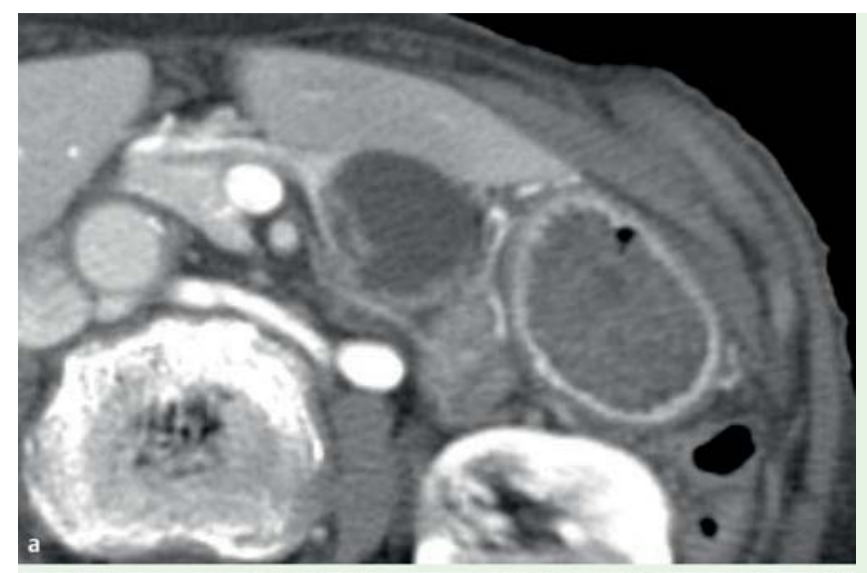

Fig. 1 Images prior to distal pancreatectomy. a Contrast-enhanced computed tomography. b Endoscopic ultrasound.
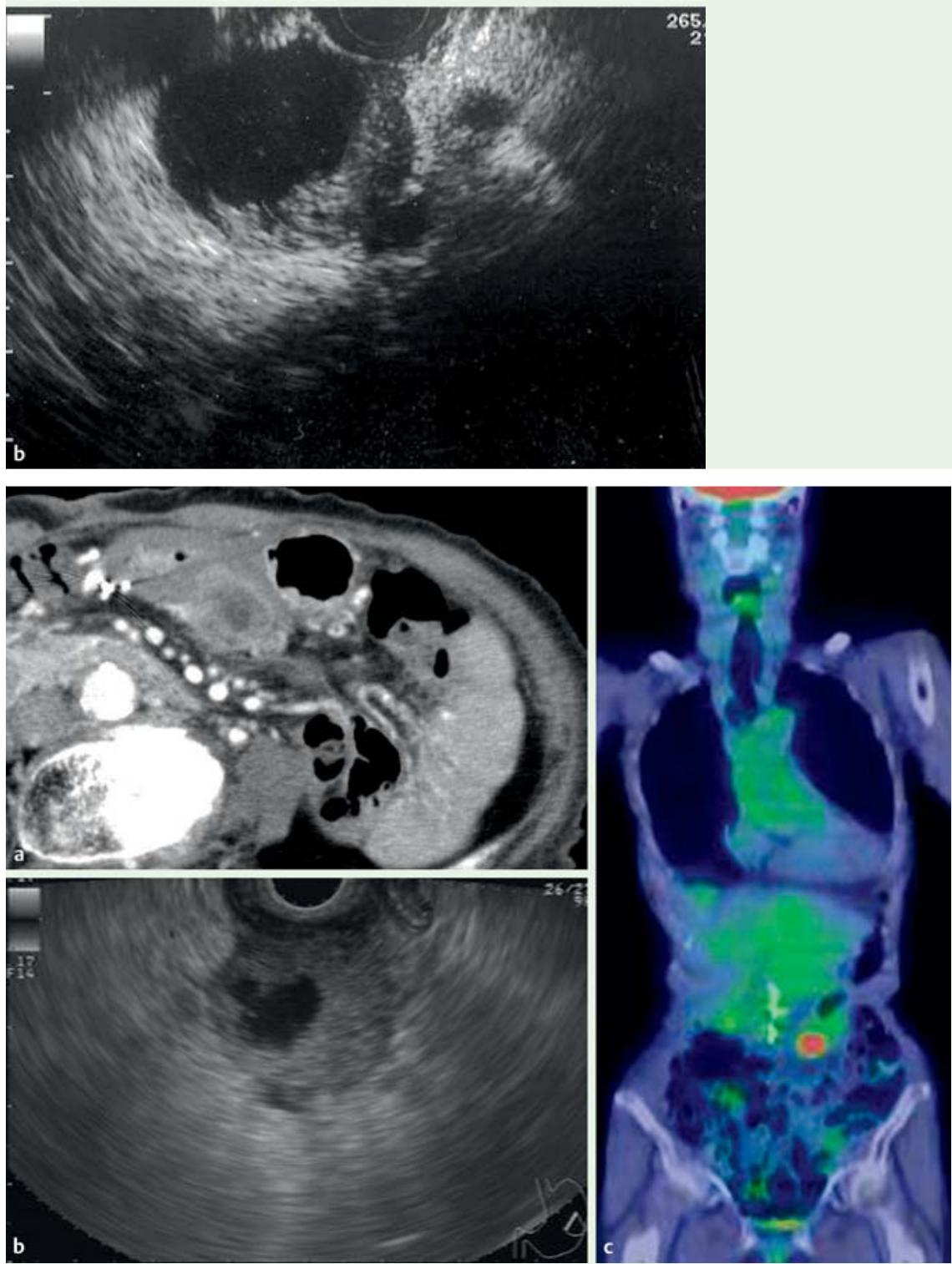

Fig.2 Images at detection of gastric metastasis. a Contrast-enhanced computed tomography. b Endoscopic ultrasound. c Positron emission tomography prior to partial gastrectomy.
An 87-year-old woman with a pancreatic mass was referred to our hospital for treatment. Contrast-enhanced computed tomography (CT) revealed a 25 -mm-diameter mass with cystic changes in the body of the pancreas ( Fig.1a). Endoscopic ultrasound-guided fine-needle aspiration (EUS-FNA) was performed using a 22gauge needle ( $\bullet$ Fig. $\mathbf{1 b}$ ), and cytohistological examination revealed a squamous cell carcinoma. Accordingly, distal pancreatectomy was performed, and the lesion was diagnosed as an adenosquamous carcinoma of the pancreas with T2NOM0 classification. The patient rejected adjuvant chemotherapy but consented to routine blood tests and contrast-enhanced CT every 3 months to monitor for recurrence.

At 19 months after surgery, the tumor marker CA19-9 level had increased within the normal range. A contrast-enhanced CT showed a 20-mm-diameter mass with cystic changes and features similar to those of the resected pancreatic mass in the posterior stomach wall, despite the absence of other metastatic lesions $(\bullet$ Fig.2a). A second EUS-FNA was performed ( $\bullet$ Fig. $\mathbf{2 b}$ ) and the lesion was identified as another squamous cell carcinoma. Positron emission tomography showed no other metastases ( $\bullet$ Fig. 2 c), and the patient underwent partial gastrectomy. The lesion was diagnosed definitely as a needle-track implantation caused by EUS-FNA, as it arose from the gastric wall subserosa ( $\bullet$ Fig. 3 a) and its histology was identical to that of the squamous cell carcinoma within the primary pancreatic carcinoma ( $\mathbf{F i g} . \mathbf{3}$ b, c). No recurrence was observed for 16 months.

To our knowledge, this is the first report of a gastric wall metastasis of pancreatic cancer caused by EUS-FNA with pathological proof of needle-track implantation. Although such a mechanism of metastasis is rare [ $1-4]$, it seems mandatory that implantation is detected before metastases occur in order to ensure radical cure. Therefore, planned examinations and cautious interpretation of blood tests and images may be essential, even following curative resection of early-stage pancreatic cancer.

Endoscopy_UCTN_Code_CPL_1AL_2AD

Competing interests: None 


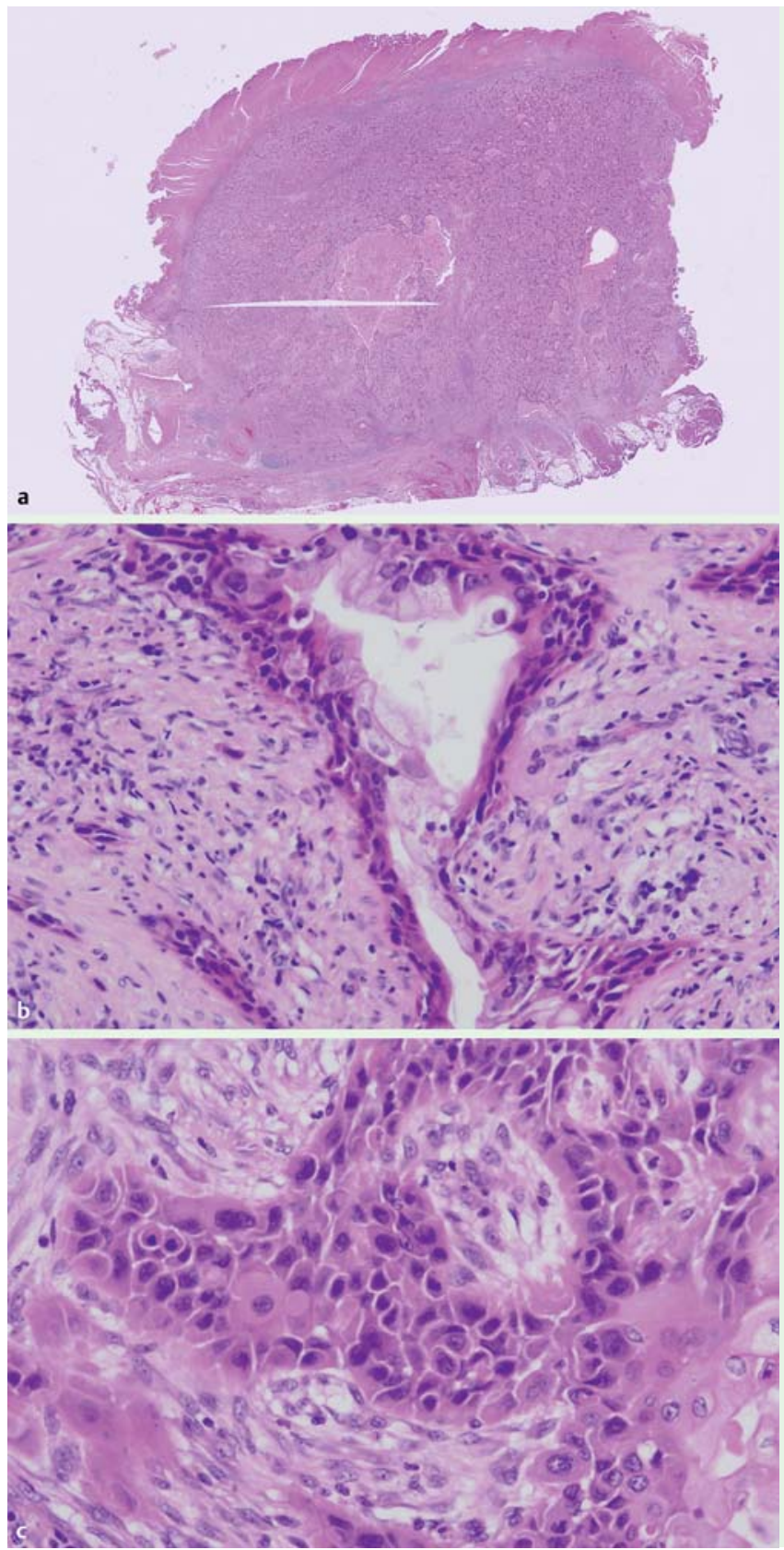

Fig. 3 Histological analysis.

a Gastric metastasis (hematoxylin and eosin [H\&E], loupe). b Gastric metastasis (H\&E × 400). c The primary pancreatic lesion $(H \& E \times 400)$.

\section{Akira Sakurada', Tsuyoshi Hayashi', Michihiro Ono ${ }^{1}$, Hirotoshi Ishiwatari ${ }^{1}$, Jiro Ogino ${ }^{2}$, Yasutoshi Kimura ${ }^{3}$, Junji Kato ${ }^{1}$}

${ }^{1}$ Department of Medical Oncology and Hematology, Sapporo Medical University School of Medicine, Sapporo, Japan

2 Department of Surgical Pathology, Sapporo Medical University School of Medicine, Sapporo, Japan

${ }^{3}$ Department of Surgery, Surgical Oncology and Science, Sapporo Medical University School of Medicine, Sapporo, Japan

\section{References}

1 Paquin SC, Gariepy G, Lepanto L et al. A first report of tumor seeding because of EUS guided FNA of a pancreatic adenocarcinoma. Gastrointest Endosc 2005; 61: 610-611

2 Chong A, Venugopal K, Segarajasingam D et al. Tumor seeding after EUS-guided FNA of pancreatic tail neoplasia. Gastrointestinal Endosc 2011; 74: 933-956

3 Katanuma A, Maguchi H, Hashigo $S$ et al. Tumor seeding after endoscopic ultrasound-guided fine-needle aspiration of cancer in the body of the pancreas. Endoscopy 2012; 44 (Suppl. 02): E160 - 161

4 Ngamruengphong $S, X u C$, Woodward TA et al. Risk of gastric or peritoneal recurrence, and long-term outcomes, following pancreatic cancer resection with preoperative endosonographically guided fine needle aspiration. Endoscopy 2013; 45: 619-626

\section{Bibliography}

DOI http://dx.doi.org/

10.1055/s-0034-1377592

Endoscopy 2015; 47: E198-E199

(c) Georg Thieme Verlag KG

Stuttgart · New York

ISSN 0013-726X

\section{Corresponding author}

\section{Tsuyoshi Hayashi, MD}

Department of Medical Oncology and Hematology Sapporo Medical University School of Medicine South-1, West-16, Chuo-ku

Sapporo 060-8543

Hokkaido

Japan

Fax: +81-11-6127987

thayashi69@sapmed.ac.jp 